\title{
New Probability Distributions in Astrophysics: V. The Truncated Weibull Distribution
}

\author{
Lorenzo Zaninetti \\ Physics Department, via P. Giuria 1, Turin, Italy \\ Email: zaninetti@ph.unito.it
}

How to cite this paper: Zaninetti, L. (2021) New Probability Distributions in Astrophys ics: V. The Truncated Weibull Distribution. International Journal of Astronomy and Astrophysics, 11, 133-149. https://doi.org/10.4236/ijaa.2021.111008

Received: January 28, 2021

Accepted: March 23, 2021

Published: March 26, 2021

Copyright (c) 2021 by author(s) and Scientific Research Publishing Inc. This work is licensed under the Creative Commons Attribution International License (CC BY 4.0).

http://creativecommons.org/licenses/by/4.0/

\begin{abstract}
We demonstrate that certain astrophysical distributions can be modelled with the truncated Weibull distribution, which can lead to some insights: in particular, we report the average value, the $r$ th moment, the variance, the median, the mode, the generation of random numbers, and the evaluation of the two parameters with maximum likelihood estimators. The first application of the Weibull distribution is the initial mass function for stars. The magnitude version of the Weibull distribution is applied to the luminosity function for the Sloan Digital Sky Survey (SDSS) galaxies and to the photometric maximum of the 2MASS Redshift Survey (2MRS) galaxies. The truncated Weibull luminosity function allows us to model the average value of the absolute magnitude as a function of the redshift for the $2 \mathrm{MRS}$ galaxies.
\end{abstract}

\section{Keywords}

Stars: Normal, Galaxy Groups, Clusters, and Superclusters, Large Scale Structure of the Universe, Cosmology

\section{Introduction}

The Weibull distribution was originally introduced to model the fracture strength of brittle and quasi-brittle materials, see [1] [2]. The Weibull distribution was successively applied to analysing the voltage breakdown of electric circuits [3] [4], the life table data of plants such as the Kolla paulula [5], the distribution of tree diameters [6], plant vegetative tissue [7] and the fatigue failure studies of human extensor digitorum longus [8]. The analysis of the truncated Weibull distribution has been explored in many papers, we list some in what follows. The upper truncated Weibull distribution has been analysed by [9] and applied to modeling component or system failure, and by [10] to modeling wind speed data and estimating wind power density. The lower truncated Weibull 
distribution has been analysed by [11]. The lower and upper truncated Weibull distribution and the evaluation of its moments have been analysed in [12] [13] [14]. A careful analysis of the above approaches allows concluding that the truncated Weibull distribution has not yet been applied to astrophysics. This paper reviews the existing Weibull distribution in Section 2, introduces the truncated Weibull distribution in Section 3, derives the Weibull luminosity function (LF) for galaxies and the connected modification due to the truncation in Section 4, and discusses, in Section 5, the application of the Weibull LF to the SDSS galaxies, to QSOs, to the photometric maximum visible in the 2MRS catalog of galaxies, and to the cosmological evolution of the average absolute magnitude with the redshift.

\section{The Weibull Distribution}

Let $X$ be a random variable defined in $[0, \infty]$; the two/parameter Weibull distribution function (DF), $F(x)$, is

$$
F(x ; b, c)=1-\mathrm{e}^{-\left(\frac{x}{b}\right)^{c}},
$$

where $b$ and $c$, both positive, are the scale and the shape parameters, see [15]. The probability density function (PDF), $f(x)$, is

$$
f(x ; b, c)=\frac{c x^{c-1} \mathrm{e}^{-\left(\frac{x}{b}\right)^{c}}}{b^{c}} .
$$

We now introduce the function

$$
\Gamma_{i}=\Gamma(1+i / c),
$$

the average value or mean, $\mu$, is

$$
\mu(b, c)=b \Gamma_{1},
$$

the variance, $\sigma^{2}$, is

$$
\sigma^{2}(b, c)=b^{2}\left(-\Gamma_{1}^{2}+\Gamma_{2}\right) \text {, }
$$

the skewness is

$$
\operatorname{skewness}(b, c)=\frac{2 \Gamma_{1}^{3}-3 \Gamma_{2} \Gamma_{1}+\Gamma_{3}}{\left(\Gamma_{1}^{2}-\Gamma_{2}\right)^{2}} \sqrt{-\Gamma_{1}^{2}+\Gamma_{2}},
$$

and the kurtosis

$$
\operatorname{kurtosis}(b, c)=-\frac{3 \Gamma_{1}^{4}-6 \Gamma_{2} \Gamma_{1}^{2}+4 \Gamma_{1} \Gamma_{3}-\Gamma_{4}}{\left(-\Gamma_{1}^{2}+\Gamma_{2}\right)^{2}} .
$$

The $r$ th moment about the origin for the Weibull distribution, $\mu_{r}^{\prime}$, is

$$
\mu_{r}^{\prime}(b, c)=b^{r} \Gamma\left(\frac{c+r}{c}\right)
$$

where $r$ is an integer and 


$$
\Gamma(z)=\int_{0}^{\infty} \mathrm{e}^{-t} t^{z-1} \mathrm{~d} t
$$

is the gamma function, see [16]. The median is at

$$
\mathrm{e}^{\frac{\ln (\ln (2))+c \ln (b)}{c}},
$$

and the mode is at

$$
\sqrt[c]{\frac{c-1}{c} b}
$$

Random generation of the Weibull variate $X$ is given by

$$
X: b, c \approx \sqrt[c]{-\ln (1-R)} b
$$

where $R$ is the unit rectangular variate. The two parameters $b$ and $c$ can be derived by the numerical solution of the two following equations which arise from the maximum likelihood estimator (MLE)

$$
\begin{gathered}
\frac{c}{b}\left(\sum_{i=1}^{n}\left(\frac{x_{i}}{b}\right)^{c}-n\right)=0, \\
-n \ln (b)+\frac{n}{c}+\sum_{i=1}^{n}-\left(\frac{x_{i}}{b}\right)^{c} \ln \left(\frac{x_{i}}{b}\right)+\ln \left(x_{i}\right)=0,
\end{gathered}
$$

where $x_{i}$ are the elements of the experimental sample with $i$ varying between 1 and $n$.

\section{The Truncated Weibull Distribution}

Let $X$ be a random variable defined in $\left[x_{l}, x_{u}\right]$; the truncated two-parameter Weibull DF, $F_{T}(x)$, is

$$
F_{T}\left(x ; b, c, x_{l}, x_{u}\right)=\frac{-\mathrm{e}^{-\left(\frac{x}{b}\right)^{c}}+\mathrm{e}^{-\left(\frac{x_{l}}{b}\right)^{c}}}{-\mathrm{e}^{-\left(\frac{x_{u}}{b}\right)^{c}}+\mathrm{e}^{-\left(\frac{x_{l}}{b}\right)^{c}}},
$$

and the PDF, $f_{T}(x)$, is

$$
f_{T}\left(x ; b, c, x_{l}, x_{u}\right)=\frac{-\left(\frac{x}{b}\right)^{c} c \mathrm{e}^{-\left(\frac{x}{b}\right)^{c}}}{x\left(\mathrm{e}^{-\left(\frac{x_{u}}{b}\right)^{c}}-\mathrm{e}^{\left.-\left(\frac{x_{l}}{b}\right)^{c}\right)}\right.},
$$

see Section 2.1 in [14].

The inequality which fixes the range of existence is $\infty>x_{u}>x>x_{l}>0$. We report the indefinite integral which characterizes the average value or mean, $\mu_{T}$,

$$
I\left(b, c, x_{l}, x_{u}, x\right)=\int x f_{T}(x ; b, c) \mathrm{d} x,
$$

which is 


$$
I\left(b, c, x_{l}, x_{u}, x\right)=\frac{I N}{I D}
$$

where

$$
\begin{aligned}
I N= & -2 c \sqrt{x} \mathrm{e}^{-\frac{1}{2} b^{-c} x^{c}+c(-\ln (x)+\ln (b))} b^{\frac{1}{2}-c}\left(2\left(\frac{1}{2}+c\right)^{2} b^{c} M_{\frac{1}{2} \frac{2 c+1}{c}, \frac{13 c+1}{2}}\left(b^{-c} x^{c}\right)\right. \\
& \left.+c\left(\left(\frac{1}{2}+c\right) b^{c}+\frac{1}{2} c x^{c}\right) M_{\frac{1}{2} c^{-1}, \frac{13 c+1}{2}}\left(b^{-c} x^{c}\right)\right),
\end{aligned}
$$

and

$$
I D=(1+c)(2 c+1)(3 c+1)\left(\mathrm{e}^{-x_{u}^{c} b^{-c}}-\mathrm{e}^{-x_{l}^{c} b^{-c}}\right)
$$

where $M_{\mu, v}(z)$ is the Whittaker $M$ function, see [17] [16]. The average value is therefore

$$
\mu\left(b, c, x_{l}, x_{u}\right)=I\left(b, c, x_{l}, x_{u}, x=x_{u}\right)-I\left(b, c, x_{l}, x_{u}, x=x_{l}\right),
$$

for a comparison, see Equation (5) in [14]. The indefinite integral which characterizes the $r$ th moment about the origin for the truncated Weibull distribution, $\mu_{r, t}^{\prime}$, is

$$
M\left(b, c, x_{l}, x_{u}, x\right)=\int x^{r} f_{T}(x ; b, c) \mathrm{d} x
$$

which is

$$
M\left(b, c, x_{l}, x_{u}, x\right)=\frac{M N}{\left(-\mathrm{e}^{-x_{u}^{c} b^{-c}}+\mathrm{e}^{-x_{l}^{c} b^{-c}}\right)(c+r)(2 c+r)(3 c+r)},
$$

where

$$
\begin{aligned}
M N= & 2 c \mathrm{e}^{-1 / 2 b^{-c} x^{c}+c(-\ln (x)+\ln (b))}\left(2 x^{r / 2} b^{r / 2}(c+r / 2)^{2} M_{1+1 / 2 \frac{r}{c}, 3 / 2+1 / 2 \frac{r}{c}}\left(b^{-c} x^{c}\right)\right. \\
& \left.+M_{1 / 2 \frac{r}{c}, 3 / 2+1 / 2 \frac{r}{c}}\left(b^{-c} x^{c}\right) c\left(1 / 2 c x^{c+r / 2} b^{r / 2-c}+b^{r / 2} x^{r / 2}(c+r / 2)\right)\right) .
\end{aligned}
$$

The $r$ th moment about the origin for the truncated Weibull distribution is therefore

$$
\mu_{r, t}^{\prime}=M\left(b, c, x_{l}, x_{u}, x=x_{u}\right)-M\left(b, c, x_{l}, x_{u}, x=x_{l}\right) .
$$

The variance, $\sigma_{T}^{2}\left(b, c, x_{l}, x_{u}\right)$, of the truncated Weibull distribution is given by

$$
\sigma_{T}^{2}\left(b, c, x_{l}, x_{u}\right)=\mu_{2, t}^{\prime}-\left(\mu_{1, t}^{\prime}\right)^{2}
$$

The median $_{T}$ in the case $x_{u}>$ median $_{T}>x_{l}$ is at

$$
\text { median }_{T}=\sqrt[c]{\left(x_{u}^{c}+x_{l}^{c}\right) b^{-c}-\ln \left(\frac{\mathrm{e}^{x_{u}^{c} b^{-c}}}{2}+\frac{\mathrm{e}^{x_{l}^{c} b^{-c}}}{2}\right)},
$$

and the mode $e_{T}$ in the case $x_{u}>\operatorname{mode}_{T}>x_{l}$ is at 


$$
\operatorname{mode} e_{T}=\sqrt[c]{\frac{c-1}{c}} b,
$$

which is the same value as that for the Weibull pdf. Random generation of the truncated Weibull variate $X$ is given by

$$
X: b, c, x_{l}, x_{u} \approx c \sqrt{\left(x_{u}^{c}+x_{l}^{c}\right) b^{-c}-\ln \left(-\mathrm{Re}^{\mathrm{x}_{u}^{c} b^{-c}}+\mathrm{Re}^{x^{c} b^{c} b^{c}}+\mathrm{e}^{x_{u}^{c} b^{c}}\right)} b,
$$

where $R$ is the unit rectangular variate. The four parameters $x_{b}, X_{l}, b$ and $c$ can be obtained in the following way. Consider a sample $\mathcal{X}=x_{1}, x_{2}, \cdots, x_{n}$ and let $x_{(1)} \geq x_{(2)} \geq \cdots \geq x_{(n)}$ denote their order statistics, so that

$x_{(1)}=\max \left(x_{1}, x_{2}, \cdots, x_{n}\right), x_{(n)}=\min \left(x_{1}, x_{2}, \cdots, x_{n}\right)$. The first two parameters $x_{l}$ and $x_{u}$ are

$$
x_{l}=x_{(n)}, \quad x_{u}=x_{(1)} .
$$

The MLE is obtained by maximizing

$$
\Lambda=\sum_{i}^{n} \ln \left(f_{T}\left(x ; b, c, x_{l}, x_{u}\right)\right) .
$$

The two derivatives $\frac{\partial \Lambda}{\partial b}=0$ and $\frac{\partial \Lambda}{\partial c}=0$ generate two non-linear equations in $b$ and $c$ which are

$$
\begin{aligned}
& \frac{N 1}{\left.-\mathrm{e}^{-\left(\frac{x_{u}}{b}\right)^{c}}+\mathrm{e}^{-\left(\frac{x_{I}}{b}\right)^{c}}\right) b}=0, \\
& \left(-\mathrm{e}^{-\left(\frac{x_{u}}{b}\right)^{c}}+\mathrm{e}^{\left.-\left(\frac{x_{I}}{b}\right)^{c}\right)}\right) c
\end{aligned}
$$

where

$$
\begin{aligned}
N 1= & \left(\left(-\mathrm{e}^{-\left(\frac{x_{u}}{b}\right)^{c}}+\mathrm{e}^{-\left(\frac{x_{l}}{b}\right)^{c}}\right) \sum_{i=1}^{n}\left(\frac{x_{i}}{b}\right)^{c}\right. \\
& \left.+\left(\left(-\left(\frac{x_{l}}{b}\right)^{c}-1\right) \mathrm{e}^{-\left(\frac{x_{l}}{b}\right)^{c}}+\mathrm{e}^{-\left(\frac{x_{u}}{b}\right)^{c}}\left(\left(\frac{x_{u}}{b}\right)^{c}+1\right)\right) n\right) c,
\end{aligned}
$$

and

$$
\begin{aligned}
N 2= & \left(-\mathrm{e}^{-\left(\frac{x_{u}}{b}\right)^{c}}+\mathrm{e}^{-\left(\frac{x_{l}}{b}\right)^{c}}\right) c \sum_{i=1}^{n}-\left(\frac{x_{i}}{b}\right)^{c} \ln \left(\frac{x_{i}}{b}\right)+\ln \left(x_{i}\right) \\
& +n\left(\left(\left(\frac{x_{I}}{b}\right)^{c} \ln \left(\frac{x_{l}}{b}\right) c-c \ln (b)+1\right) \mathrm{e}^{-\left(\frac{x_{I}}{b}\right)^{c}}\right. \\
& \left.-\mathrm{e}^{-\left(\frac{x_{u}}{b}\right)^{c}}\left(\left(\frac{x_{u}}{b}\right)^{c} \ln \left(\frac{x_{u}}{b}\right) c-c \ln (b)+1\right)\right) .
\end{aligned}
$$




\section{The luminosity Function}

This section reports the luminosity functions (LFs) for the Weibull distribution and the truncated Weibull distribution.

\subsection{The Weibull LF}

The Schechter function, introduced by [18], provides a useful reference for the LF of galaxies

$$
\Phi\left(L ; \alpha, L^{*}, \Phi^{*}\right) \mathrm{d} L=\left(\frac{\Phi^{*}}{L^{*}}\right)\left(\frac{L}{L^{*}}\right)^{\alpha} \exp \left(-\frac{L}{L^{*}}\right) \mathrm{d} L,
$$

here $\alpha$ sets the slope for low values of $L, L^{*}$ is the characteristic luminosity and $\Phi^{*}$ is the normalization. The equivalent distribution in absolute magnitude is

$$
\Phi(M) \mathrm{d} M=0.921 \Phi^{*} 10^{0.4(\alpha+1)\left(M^{*}-M\right)} \exp \left(-10^{0.4\left(M^{*}-M\right)}\right) \mathrm{d} M
$$

where $M^{*}$ is the characteristic magnitude as derived from the data. We now introduce the parameter $h$, which is $H_{0} / 100$, where $H_{0}$ is the Hubble constant. The scaling with $h$ is $M^{*}-5 \log _{10} h$ and $\Phi^{*} h^{3}\left[\mathrm{Mpc}^{-3}\right]$. In order to derive the Weibull LF we start from the PDF as given by Equation (2),

$$
\Psi\left(L ; c, L^{*}, \Psi^{*}\right) \mathrm{d} L=\Psi^{*} \frac{\left(\frac{L}{L^{*}}\right)^{c} c \mathrm{e}^{-\left(\frac{L}{L^{*}}\right)^{c}}}{L} \mathrm{~d} L,
$$

where $L$ is the luminosity, $L^{*}$ is the characteristic luminosity and $\Psi^{*}$ is the normalization and the version in absolute magnitude is

$$
\Psi\left(M ; c, M^{*}, \Psi^{*}\right) \mathrm{d} M=0.4 \Psi^{*} 10^{\left(-0.4 M+0.4 M^{*}\right) c} c \mathrm{e}^{-10^{\left(-0.4 M+0.4 M^{*}\right) c}} \ln (10) \mathrm{d} M .
$$

\subsection{The Truncated Weibull LF}

We start with the truncated Weibull PDF with scaling as given by Equation (15)

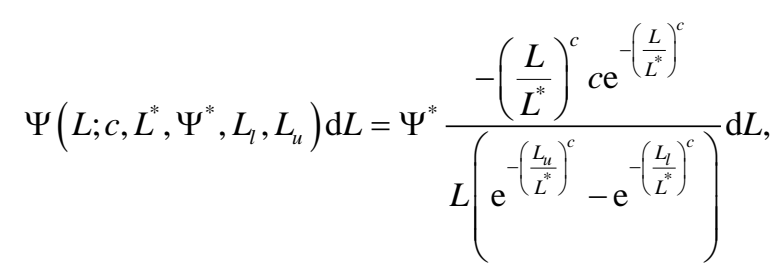

where $L$ is the luminosity, $L^{*}$ is the characteristic luminosity, $L_{l}$ is the lower boundary in luminosity, $L_{u}$ is the upper boundary in luminosity, and $\Psi^{*}$ is the normalization. The magnitude version is

$$
\begin{aligned}
& \Psi\left(M ; c, M^{*}, \Psi^{*}, M_{l}, M_{u}\right) \mathrm{d} M \\
& =\Psi^{*} \frac{-0.4\left(10^{0.4 M^{*}-0.4 M}\right)^{c} c \mathrm{e}^{-\left(10^{0.4 M^{*}-0.4 M}\right)^{c}}(\ln (2)+\ln (5))}{\mathrm{e}^{-\left(10^{-0.4 M_{l}+0.4 M^{*}}\right)^{c}}-\mathrm{e}^{-\left(10^{0.4 M^{*}-0.4 M_{u}}\right)^{c}} \mathrm{~d} M}
\end{aligned}
$$


where $M$ is the absolute magnitude, $M^{*}$ the characteristic magnitude, $M_{l}$ the lower boundary in magnitude, $M_{u}$ the upper boundary in magnitude and $\Psi^{*}$ is the normalization. The mean theoretical absolute magnitude, $\langle M\rangle$, can be evaluated as

$$
\langle M\rangle=\frac{\int_{M_{l}}^{M_{u}} M \times \Psi\left(M ; c, M^{*}, \Psi^{*}, M_{l}, M_{u}\right) \mathrm{d} M}{\int_{M_{l}}^{M_{u}} \Psi\left(M ; c, M^{*}, \Psi^{*}, M_{l}, M_{u}\right) \mathrm{d} M} .
$$

\section{Astrophysical Applications}

This section reviews the adopted statistics, applies the truncated Weibull distribution to the initial mass function (IMF) for stars, models the LF for galaxies and QSOs, explains the photometric maximum in the number of galaxies of the 2MRS, and traces the cosmological evolution of the average absolute magnitude.

\subsection{Statistics}

The merit function $\chi^{2}$ is computed according to the formula

$$
\chi^{2}=\sum_{i=1}^{n} \frac{\left(T_{i}-O_{i}\right)^{2}}{T_{i}}
$$

where $n$ is the number of bins, $T_{i}$ is the theoretical value, and $O_{i}$ is the experimental value represented by the frequencies. The theoretical frequency distribution is given by

$$
T_{i}=N \Delta x_{i} p(x),
$$

where $N$ is the number of elements of the sample, $\Delta x_{i}$ is the magnitude of the size interval, and $p(x)$ is the PDF under examination.

A reduced merit function $\chi_{\text {red }}^{2}$ is given by

$$
\chi_{\text {red }}^{2}=\chi^{2} / N F \text {, }
$$

where $N F=n-k$ is the number of degrees of freedom, $n$ is the number of bins, and $k$ is the number of parameters. The goodness of the fit can be expressed by the probability $Q$, see equation 15.2.12 in [19], which involves the number of degrees of freedom and $\chi^{2}$. According to [19] p. 658, the fit may be acceptable' if $Q>0.001$.

The Akaike information criterion (AIC), see [20], is defined by

$$
\mathrm{AIC}=2 k-2 \ln (L),
$$

where $L$ is the likelihood function and $k$ the number of free parameters in the model. We assume a Gaussian distribution for the errors. Then the likelihood function can be derived from the $\chi^{2}$ statistic $L \propto \exp \left(-\frac{\chi^{2}}{2}\right)$ where $\chi^{2}$ has been computed by Equation (41), see [21], [22]. Now the AIC becomes

$$
\mathrm{AIC}=2 k+\chi^{2} .
$$

The Kolmogorov-Smirnov test (K-S), see [23] [24] [25], does not require bin- 
ning the data. The K-S test, as implemented by the FORTRAN subroutine KSONE in [19], finds the maximum distance, $D$, between the theoretical and the astronomical $\mathrm{CDF}$ as well the significance level $P_{K S}$, see formulas 14.3 .5 and 14.3.9 in [19]; if $P_{K S} \geq 0.1$, the goodness of the fit is believable.

\subsection{The IMF for Stars}

We tested the truncated Weibull distribution on four samples of stars: NGC 2362 (271 stars), the young cluster NGC 6611 (207 stars), the $\gamma$ Velorum cluster (237 stars), and the young cluster Berkeley 59 (420 stars), for more details, see Section 5.2 of [26]. The results are presented in Table 1 for the truncated Weibull distribution with two parameters, where the last column reports whether the results are better compared to the lognormal distribution $(\mathrm{Y})$ or worse $(\mathrm{N})$. Results on the lognormal distribution were reported in Table 1 in [26].

Graphical displays of the empirical PDF visualized through histograms as well as the theoretical PDF for NGC 6611 are reported in Figure 1 and those for the $\gamma$ Velorum sample are reported in Figure 2.

\subsection{The LF for Galaxies}

A test has been performed on the $u^{*}$ band of SDSS as in [27] with data available at https://cosmo.nyu.edu/blanton/lf.html. The Schechter function, the new Weibull LF represented by formula (37) and the data are reported in Figure 3, parameters as in Table 2.

A careful examination of Table 2 reveals that the Weibull LF has a lower $\chi_{\text {red }}^{2}$ compared to the Schechter LF.

Another case is the LF for QSO in the case $0.3<z<0.5$, see [28] for more details. Figure 4 displays the observed LF for QSO as well the theoretical fit with the Weibull LF. The parameters and the statistical results for the Schechter LF are reported in Table 3 and those for the Weibull LF in Table 4; the Weibull LF

Table 1. Numerical values of $\chi_{\text {red }}^{2}$, AIC, probability $Q, D$, the maximum distance between theoretical and observed DF, and $P_{K S}$, significance level, in the K-S test of the truncated Weibull distribution with two parameters for different mass distributions. The last column (LN) indicates an AIC lower $(\mathrm{Y})$ or bigger $(\mathrm{N})$ in respect to the lognormal distribution. The number of linear bins, $n$, is 20 .

\begin{tabular}{|c|c|c|c|c|c|c|c|}
\hline Cluster & parameters & AIC & $\chi_{\text {red }}^{2}$ & $Q$ & $D$ & $P_{K S}$ & $\mathrm{LN}$ \\
\hline NGC 2362 & $\begin{array}{ll}b=0.726, & c=2.2, \\
x_{l}=0.12, & x_{u}=1.47\end{array}$ & 39.5 & 1.96 & 0.011 & 0.011 & 0.576 & $\mathrm{~N}$ \\
\hline NGC 6611 & $\begin{array}{l}b=0.483, \quad c=1.011, \\
x_{l}=0.019, \quad x_{u}=1.46\end{array}$ & 47.77 & 2.48 & $8.4 \times 10^{-4}$ & 0.059 & 0.45 & $\mathrm{Y}$ \\
\hline$\gamma$ Velorum & $\begin{array}{cc}b=0.153, & c=0.745 \\
x_{l}=0.158, & x_{u}=1.317\end{array}$ & 31.24 & 1.45 & 0.107 & 0.063 & 0.292 & $\mathrm{Y}$ \\
\hline Berkeley 59 & $\begin{array}{ll}b=0.347, & c=1.143, \\
x_{l}=0.16, & x_{u}=2.24\end{array}$ & 83.71 & 4.73 & $9.74 \times 10^{-10}$ & 0.122 & $6.35 \times 10^{-6}$ & $\mathrm{~N}$ \\
\hline
\end{tabular}




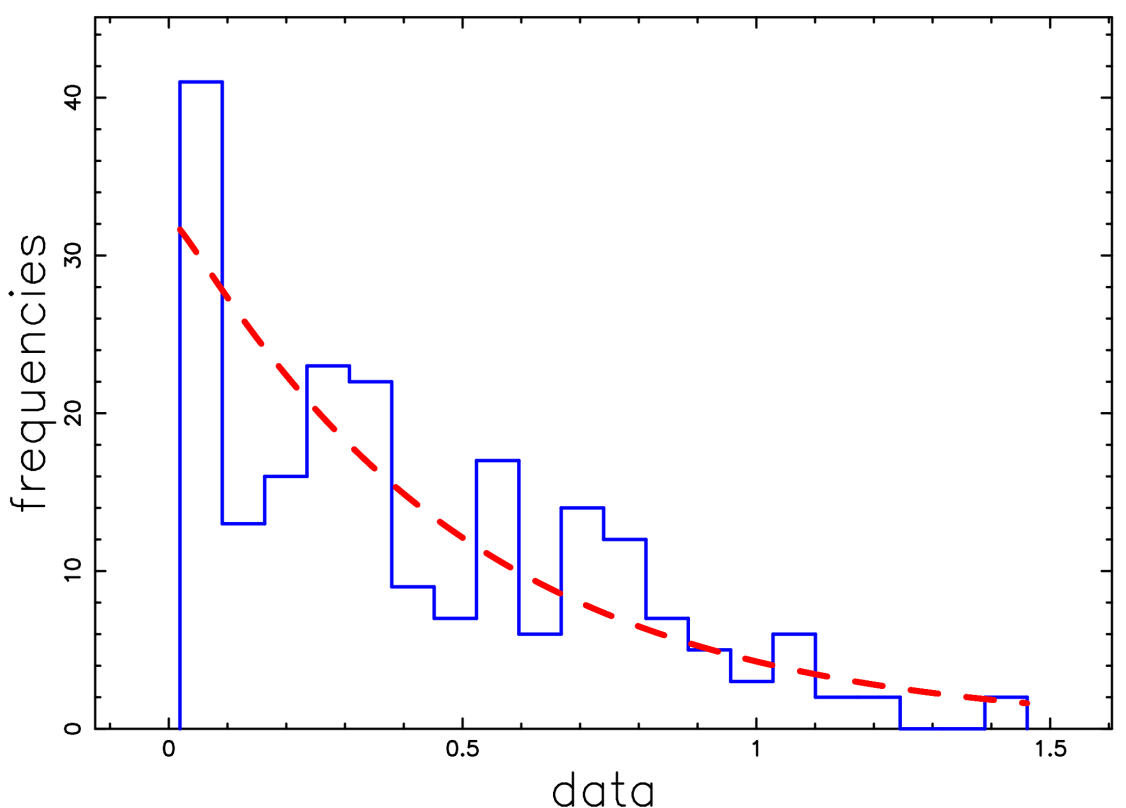

Figure 1. Empirical PDF of the mass distribution for NGC 6611 cluster data (blue histogram) with a superposition of the truncated Weibull PDF (red line). Theoretical parameters as in Table 1.

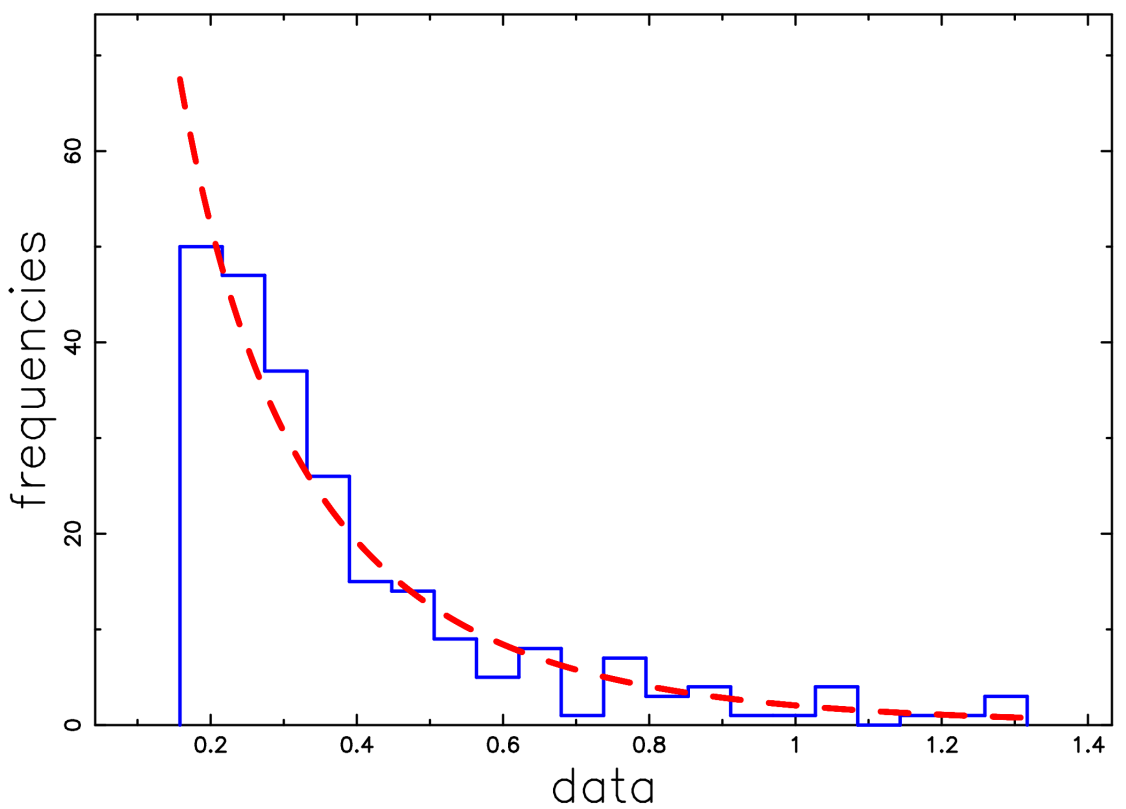

Figure 2. Empirical PDF of the mass distribution for $\gamma$ Velorum data (blue histogram) with a superposition of the truncated Weibull PDF (red line). Theoretical parameters as in Table 1.

Table 2. Numerical values and $\chi_{\text {red }}^{2}$ of the LFs applied to SDSS Galaxies in the $u^{*}$ band.

\begin{tabular}{ccc}
\hline LF & parameters & $\chi_{\text {red }}^{2}$ \\
\hline Schechter & $M^{*}=-17.92$, & $\alpha=-0.9, \quad \Phi^{*}=0.03 / \mathrm{Mpc}^{3}$ \\
Weibull & $M^{*}=-16.69, \quad c=0.728, \quad \Psi^{*}=0.0718 / \mathrm{Mpc}^{3}$ & 0.689 \\
\hline
\end{tabular}




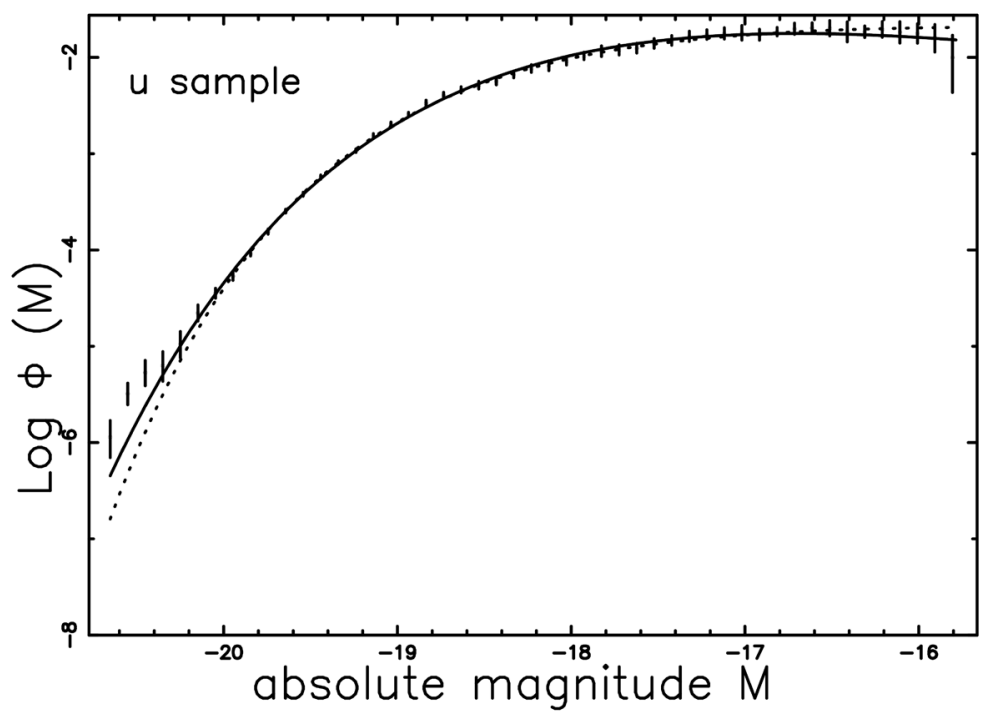

Figure 3. The LF data of SDSS $\left(u^{*}\right)$ are represented with error bars. The continuous line fit represents the Weibull LF (37) and the dotted line represents the Schechter function.

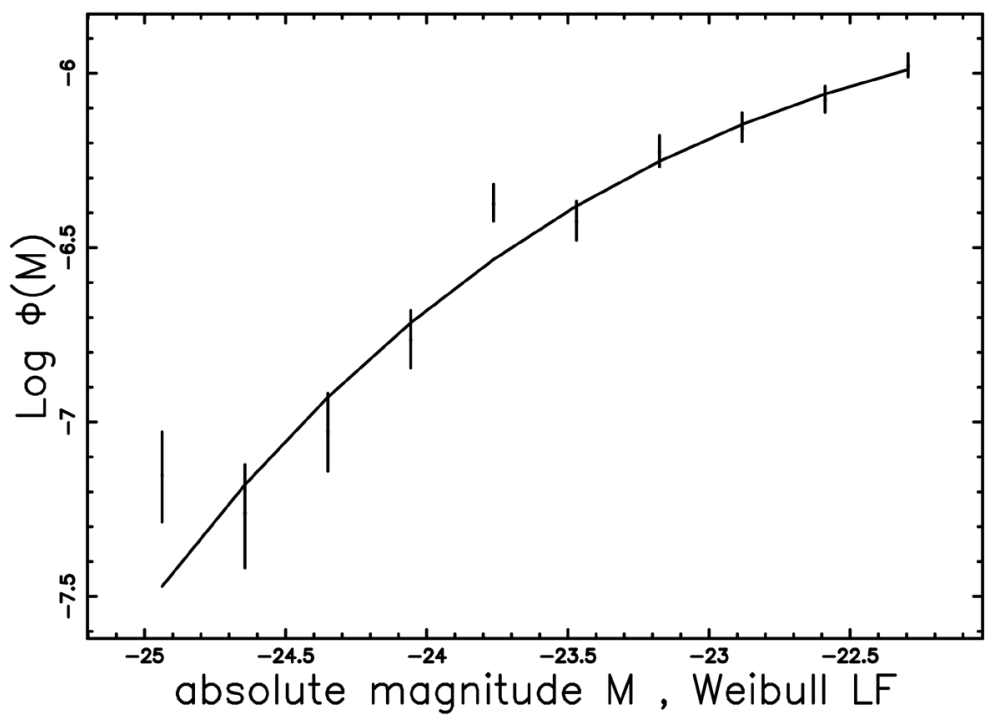

Figure 4. The observed LF for QSOs, empty stars with error bar, and the fit by the Weibull LF for $z$ in $[0.3,0.5]$ and $M$ in $[-24.93,-22]$. Parameters as in Table 4.

Table 3. Parameters of the Schechter LF in the range of redshift $[0.3,0.5]$ when $k=3$ and $n=10$

\begin{tabular}{ccccccc}
\hline$M^{*}$ & $\Psi^{*}$ & $\alpha$ & $\chi^{2}$ & $\chi_{\text {red }}^{2}$ & $Q$ & AIC \\
\hline-23.75 & $8.85 \times 10^{-7}$ & -1.37 & 10.49 & 1.49 & 0.162 & 16.49 \\
\hline
\end{tabular}

Table 4. Parameters of the Weibull LF for QSOs in the range of redshift $[0.3,0.5]$ when $k=3$ and $n=10$.

\begin{tabular}{ccccccc}
\hline$M^{*}$ & $\Psi^{*}$ & $\alpha$ & $\chi^{2}$ & $\chi_{\text {red }}^{2}$ & $Q$ & AIC \\
\hline-20.566 & $9.26 \times 10^{-6}$ & 0.471 & 10.08 & 1.44 & 0.183 & 16.08 \\
\hline
\end{tabular}


has smaller $\chi_{\text {red }}^{2}$ compared to the Schechter LF.

\subsection{The Photometric Maximum}

In the pseudo-Euclidean universe, the correlation between the expansion velocity and distance is

$$
V=H_{0} D=c_{l} z,
$$

where $H_{0}$ is the Hubble constant, $H_{0}=100 \mathrm{~h} \mathrm{~km} \cdot \mathrm{s}^{-1} \cdot \mathrm{Mpc}^{-1}$, with $h=1$ when $h$ is not specified, $D$ is the distance in Mpc, $c_{l}$ is the speed of light and $z$ is the redshift. In the pseudo-Euclidean universe, the flux of radiation, $f$, expressed in units of $\frac{L_{\odot}}{\mathrm{Mpc}^{2}}$, where $L_{\odot}$ represents the luminosity of the sun, is

$$
f=\frac{L}{4 \pi D^{2}},
$$

where $D$ represents the distance of the galaxy expressed in $\mathrm{Mpc}$, and

$$
D=\frac{C_{l} Z}{H_{0}} .
$$

The joint distribution in $z$ and $f$ for a generic LF, $\Phi\left(\frac{z^{2}}{z_{\text {crit }}^{2}}\right)$ is

$$
\frac{\mathrm{d} N}{\mathrm{~d} \Omega \mathrm{d} z \mathrm{~d} f}=4 \pi\left(\frac{c_{l}}{H_{0}}\right)^{5} z^{4} \Phi\left(\frac{z^{2}}{z_{c r i t}^{2}}\right),
$$

where $\mathrm{d} \Omega, \mathrm{d} z$ and $\mathrm{d} f$ represent the differentials of the solid angle, the redshift, and the flux, respectively, and

$$
z_{\text {crit }}^{2}=\frac{H_{0}^{2} L^{*}}{4 \pi f c_{l}^{2}}
$$

where $L^{*}$ is the characteristic luminosity, for more details, see [29]. The LF is chosen to be the Schechter function, but different LFs can be tested, for example, the Weibull LF. The joint distribution in $z$, fand $\Omega$ for galaxies for the Weibull LF, see Equation (36), is

$$
\frac{\mathrm{d} N\left(z ; c, \Psi^{*}, z_{\text {crit }}\right)}{\mathrm{d} \Omega \mathrm{d} z \mathrm{~d} f}=\frac{4 z^{2} c_{l}^{5} \Psi^{*}\left(\frac{z^{2}}{z_{\text {crit }}^{2}}\right)^{c} c \pi \mathrm{z}_{\text {crit }}^{2} \mathrm{e}^{-\left(\frac{z^{2}}{z_{\text {crit }}^{2}}\right)^{c}}}{H_{0}^{5} L^{*}} .
$$

The above number of galaxies in $z$ and $f$ has a maximum at $z=z_{\max }$ which is the solution of the following non-linear equation

$$
-8 z c_{l}^{5} \Psi\left(\frac{z^{2}}{z_{\text {crit }}^{2}}\right)^{c} c \pi z_{\text {crit }}^{2} \mathrm{e}^{-\left(\frac{z^{2}}{z_{\text {crit }}^{2}}\right)^{c}}\left(\left(\frac{z^{2}}{z_{\text {crit }}^{2}}\right)^{c} c-c-1\right)=0 .
$$

A first numerical evaluation of the position in $z$ of the above equation is reported in units of $z_{c r i t}$, see the blue dashed line in Figure 5. A second analytical result can be obtained inserting for the number of galaxies a numerical value for 


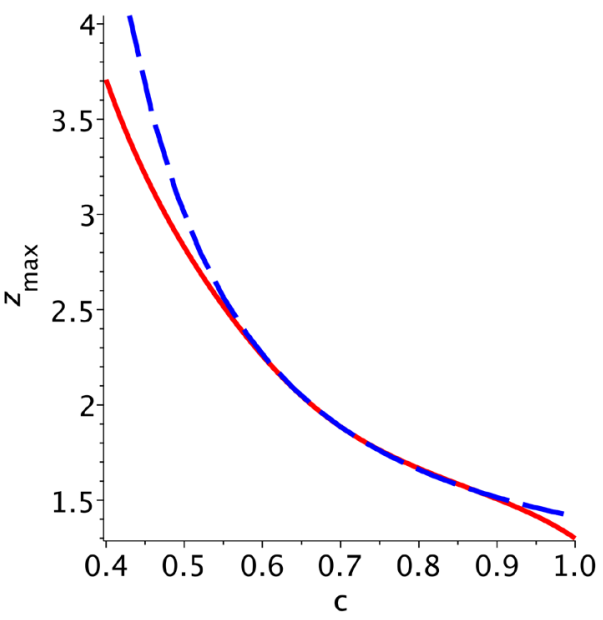

Figure 5. Position in units of $z_{c r i t}$ of the photometric maximum as a function of the shape parameter $c$ numerical solution (blue dashed line) and Taylor solution (red full line).

c. As an example when $c=1 / 2$, the nonlinear equation for the photometric maximum is

$$
-2 z c_{l}^{5} \Psi^{*} \sqrt{\frac{z^{2}}{z_{\text {crit }}^{2}}} \pi z_{\text {crit }}^{2} \mathrm{e}^{-\sqrt{\frac{z^{2}}{z_{\text {crit }}^{2}}}}\left(\sqrt{\frac{z^{2}}{z_{\text {crit }}^{2}}}-3\right)=0,
$$

which has a physical solution at

$$
z_{\max }=3 z_{\text {crit }} .
$$

A third approximate result is obtained using a Taylor expansion of Equation (52) around $z=2 z_{\text {crit }}$ of order 3 , which gives

$$
\begin{aligned}
& z_{\max }=z_{c r i t} \times \\
& \frac{2464^{c} c^{3}-28 c^{3} 16^{c}+2^{2 c+2} c^{3}-4 c^{3} 256^{c}+464^{c} c^{2}-12 c^{2} 16^{c}+2^{2 c+2} c^{2}+c 16^{c}-c 4^{c}-A-4^{c}}{c\left(1264^{c} c^{2}-2 c^{2} 256^{c}-14 c^{2} 16^{c}+2 c^{2} 4^{c}+3 c 64^{c}-9 c 16^{c}+3 c 4^{c}-16^{c}+4^{c}\right)}, \\
& \text { where } \\
& \qquad \begin{aligned}
A= & -4 c^{4} 16^{c}+40 \times 64^{c} c^{4}+32 \times 1024^{c} c^{4}+56 \times 64^{c} c^{3}-48 c^{3} 256^{c} \\
& -8 c^{3} 16^{c}+14 \times 64^{c} c^{2}-3 c^{2} 16^{c}+2 c 16^{c}-3 c^{2} 256^{c}-2 c 64^{c} \\
& \left.+8 \times 1024^{c} c^{3}-60 \times 256^{c} c^{4}-4 \times 4096^{c} c^{4}+16^{c}\right)^{1 / 2} .
\end{aligned}
\end{aligned}
$$

A graphical display of the Taylor solution is reported in Figure 5 as the red full line. Figure 6 reports the number of observed galaxies for the 2MASS Redshift Survey (2MRS) catalog at a given apparent magnitude and both the Schechter and the Weibull models for the number of galaxies as functions of the redshift. The influence on the above curve of varying $M^{*}$ is reported in Figure 7.

The mean redshift for galaxies $\langle z\rangle$ is

$$
\langle z\rangle=\frac{\int_{0}^{\infty} z \frac{\mathrm{d} N}{\mathrm{~d} \Omega \mathrm{d} z \mathrm{~d} f} \mathrm{~d} z}{\int_{0}^{\infty} \frac{\mathrm{d} N}{\mathrm{~d} \Omega \mathrm{d} z \mathrm{~d} f} \mathrm{~d} z} .
$$




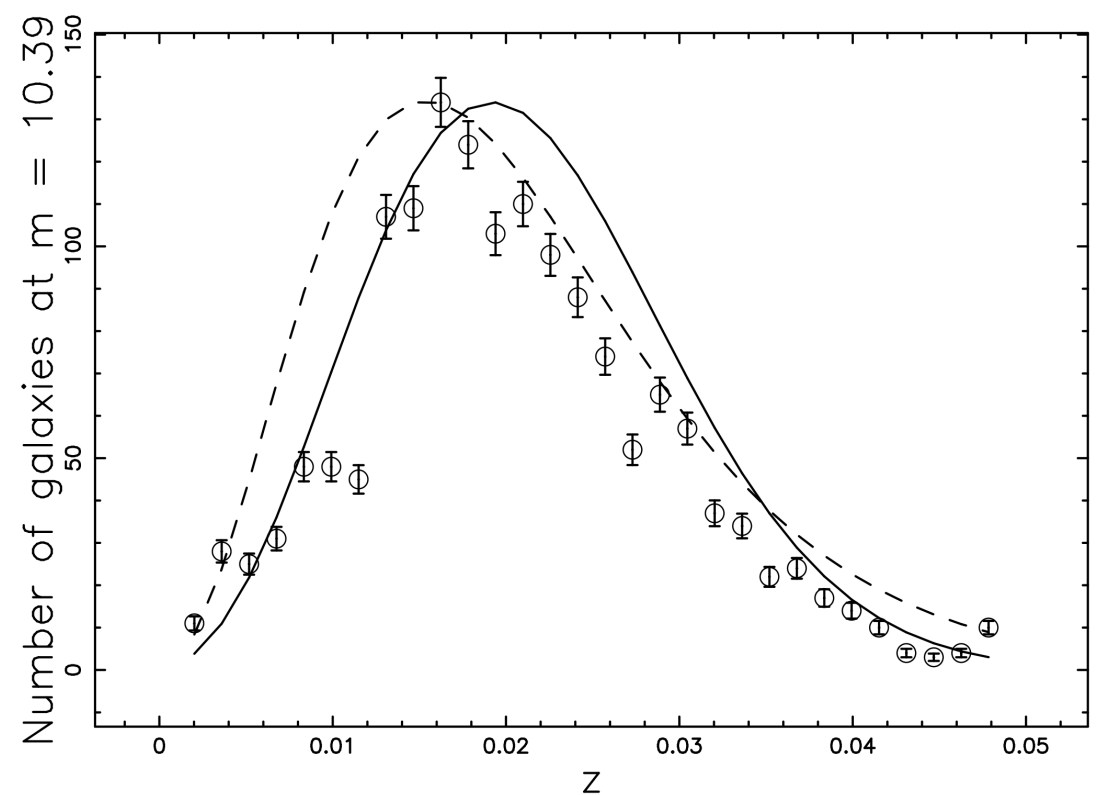

Figure 6. The galaxies of the 2 MRS with $10.31 \leq m \leq 10.47$ or $1164793 \frac{L_{\odot}}{\mathrm{Mpc}^{2}} \leq f \leq 1346734 \frac{L_{\odot}}{\mathrm{Mpc}^{2}}$ are organized in frequencies versus heliocentric redshift, (empty circles); the error bar is given by the square root of the frequency. The maximum frequency of observed galaxies is at $z=0.017$. The full line is the theoretical curve generated by $\frac{\mathrm{d} N}{\mathrm{~d} \Omega \mathrm{d} z \mathrm{~d} f}(z)$ as given by the application of the Schechter LF which is Equation (43) in [29] and the dashed line represents the Weibull LF which is Equation (51). The Weibull LF parameters are $c=1 / 2$ and $M^{*}=-20.65, \chi^{2}=198$ for the Schechter LF and $\chi^{2}=452$ for the Weibull LF.

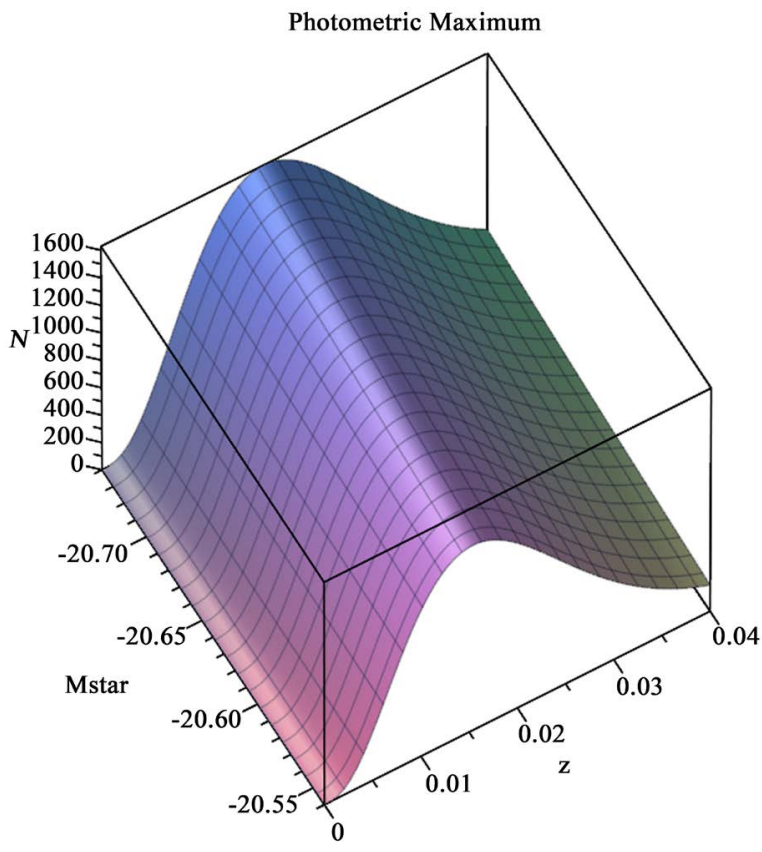

Figure 7. The theoretical number of galaxies for the Weibull LF as function of the redshift and $M^{*}$; parameters as in Figure 6. 
The mean redshift for the Weibull LF as a function of $z_{c r i t}$ when $c=1 / 2$ is

$$
\langle z\rangle\left(z_{\text {crit }}\right)=4 z_{\text {crit }} \quad \text { when } c=1 / 2 \text {, }
$$

or as a function of the flux

$$
\langle z\rangle(f)=\frac{2 \sqrt{\pi f 10^{0.4 M_{\odot}-0.4 M^{*}}} H_{0}}{\pi f c_{l}} \quad \text { when } c=1 / 2,
$$

where $M_{\odot}=3.39$ is the reference magnitude of the sun at the considered bandpass, or as a function of the apparent magnitude

$$
\langle z\rangle(m)=\frac{4 \times 10^{-5} \sqrt{\mathrm{e}^{0.921 M_{\odot}-0.921 m} 10^{0.4 M_{\odot}-0.4 M^{*}}} H_{0}}{\mathrm{e}^{0.921 M_{\odot}-0.921 m} c_{l}} \text { when } c=1 / 2 \text {. }
$$

\subsection{Mean Absolute Magnitude}

The absolute magnitude which can be observed as a function of the limiting apparent magnitude, $m_{L}$, is

$$
M_{L}=m_{L}-5 \log _{10}\left(\frac{C Z}{H_{0}}\right)-25,
$$

where $m_{L}=11.75$ for the 2 MRS catalog.

The theoretical average absolute magnitude of the truncated Weibull LF, see Equation (40), can be compared with the observed average absolute magnitude of the $2 \mathrm{MRS}$ as a function of the redshift. To fit the data, we assumed the following empirical dependence on the redshift for the characteristic magnitude of the truncated Weibull LF.

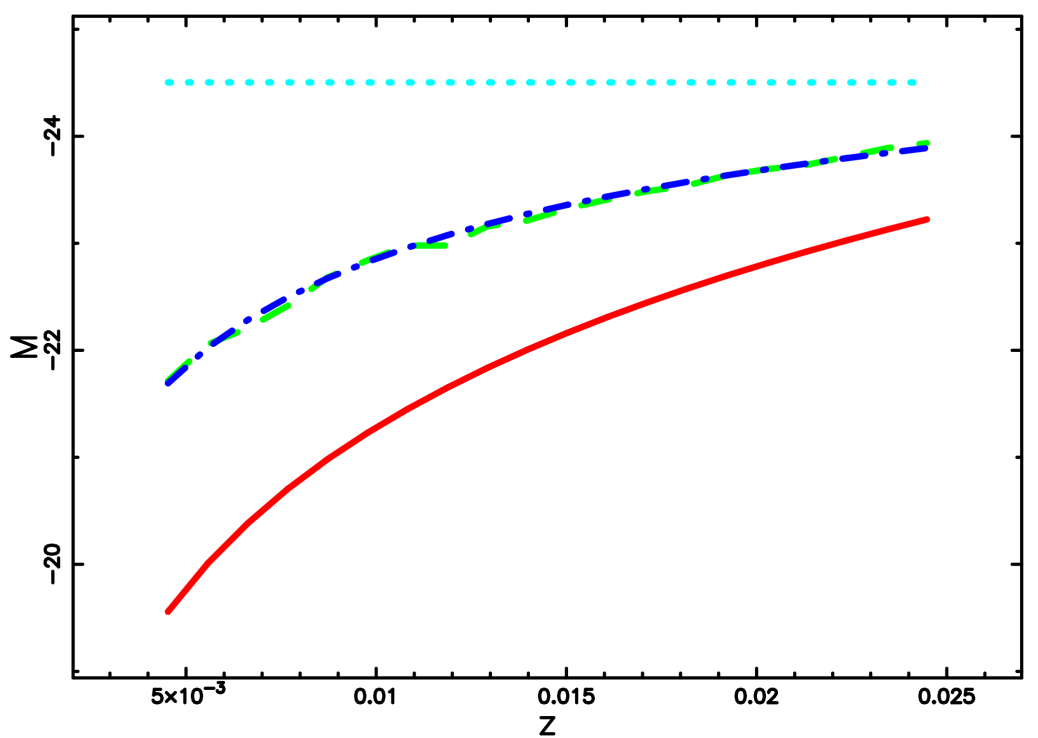

Figure 8. Average absolute magnitude of the galaxies belonging to the $2 \mathrm{MRS}$ (green-dashed line), theoretical average absolute magnitude for the truncated Weibull LF (blue dash-dot-dash-dot line) as given by equation (42), lower theoretical curve as represented by Equation (63) (red line) and minimum absolute magnitude observed (cyan dotted line). 


$$
M^{*}=-25.14+4\left(1-\left(\frac{Z-Z_{\min }}{Z_{\max }-z_{\min }}\right)^{0.7}\right) \text {. }
$$

This relationship models the decrease of the characteristic absolute magnitude as a function of the redshift and allows us to match the observational and theoretical data. The lower bound in absolute magnitude is given by the minimum magnitude of the selected bin, the upper bound is given by Equation (61), the characteristic magnitude varies according to Equation (62) and Figure 8 reports a comparison between the theoretical and the observed absolute magnitude for the $2 \mathrm{MRS}$ catalog.

\section{Conclusions}

\section{Truncated Weibull distribution}

We derived the PDF, the DF, the average value, the $r$ th moment, the variance, the median, the mode, an expression to generate random numbers and the way to obtain the two parameters, $b$ and $c$, by the MLE for the truncated Weibull distribution.

\section{Weibull luminosity function}

We derived the Weibull LF in the standard and the truncated case: the application to both the SDSS Galaxies and to the QSOs in the range of redshift $[0.3,0.5]$ yields a lower reduced merit function compared to Schechter LF, see Table 2 and Table 4.

\section{Cosmological applications}

The number of galaxies as functions of the redshift, the flux and the solid angle for the Weibull LF in the pseudo-Euclidean universe presents a maximum which can be compared with the observed one for the 2MRS, see Figure 6. The truncated Weibull LF produces a good fit to the average absolute magnitude of the $2 \mathrm{MRS}$ galaxies as a function of the redshift, see Figure 8 .

\section{Conflicts of Interest}

The author declares no conflicts of interest regarding the publication of this paper.

\section{References}

[1] Weibull, W. (1939) A Statistical Theory of Strengths of Materials. Ingeniörsvetenskapsakademiens handlingar, No. 151, Generalstabens litografiska anstalts förlag, Stockholm.

[2] Weibull, W. (1951) A Statistical Distribution Function of Wide Applicability. Journal of Applied Mechanics, 18, 293-297.

[3] Cacciari, M., Mazzanti, G. and Montanari, G. (1996) Comparison of Maximum Likelihood Unbiasing Methods for the Estimation of the Weibull Parameters. IEEE Transactions on Dielectrics and Electrical Insulation, 3, 18. https://doi.org/10.1109/94.485511

[4] Hirose, H. (1996) Maximum Likelihood Estimation in the 3-Parameter Weibull Distribution. A Look through the Generalized Extreme-Value Distribution. IEEE 
Transactions on Dielectrics and Electrical Insulation, 3, 43-55. https://doi.org/10.1109/94.485513

[5] Tuan, S.J., Chang, P.W., Saska, P., Atlihan, R. and Chi, H. (2017) Host Plants Mixture and Fitness of Kolla paulula: With an Evaluation of the Application of Weibull Function. Journal of Applied Entomology, 141, 329-338. https://doi.org/10.1111/jen.12357

[6] Diamantopoulou, M.J., Özçelik, R., Crecente-Campo, F. and Eler, Ü. (2015) Estimation of Weibull Function Parameters for Modelling Tree Diameter Distribution Using Least Squares and Artificial Neural Networks Methods. Biosystems Engineering, 133, 33-45. https://doi.org/10.1016/j.biosystemseng.2015.02.013

[7] Pitt, R. (1982) Models for the Rheology and Statistical Strength of Uniformly Stressed Vegetative Tissue. Transactions of the ASAE, 25, 1776-1784.

[8] Gallagher, S. (2012) Weibull Analyses of the Fatigue Life of Human Tissues. Proceedings of the Human Factors and Ergonomics Society Annual Meeting, Vol. 56, 1130-1134. https://doi.org/10.1177/1071181312561246

[9] Zhang, T. and Xie, M. (2011) On the Upper Truncated Weibull Distribution and Its Reliability Implications. Reliability Engineering \& System Safety, 96, 194-200. https://doi.org/10.1016/j.ress.2010.09.004

[10] Kantar, Y.M. and Usta, I. (2015) Analysis of the Upper-Truncated Weibull Distribution for Wind Speed. Energy Conversion and Management, 96, 81-88. https://doi.org/10.1016/j.enconman.2015.02.063

[11] Wingo, D.R. (1989) The Left-Truncated Weibull Distribution: Theory and Computation. Statistical Papers, 30, 39-48. https://doi.org/10.1007/BF02924307

[12] McEwen, R.P. and Parresol, B.R. (1991) Moment Expressions and Summary Statistics for the Complete and Truncated Weibull Distribution. Communications in Statistics - Theory and Methods, 20, 1361-1372. https://doi.org/10.1080/03610929108830570

[13] Khan, R., Anwar, Z. and Athar, H. (2007) Recurrence Relations for Single and Product Moments of Generalized Order Statistics from Doubly Truncated Weibull Distribution. Aligarh Journal of Statistics, 27, 69-79.

[14] Crénin, F. (2015) Truncated Weibull Distribution Functions and Moments. https://doi.org/10.2139/ssrn.2690255

[15] Forbes, C., Evans, M., Hastings, N. and Peacock, B. (2011) Statistical Distributions. Fourth Edition, John Wiley \& Sons, Hoboken. https://doi.org/10.1002/9780470627242

[16] Olver, F.W.J., Lozier, D.W., Boisvert, R.F. and Clark, C.W. (2010) NIST Handbook of Mathematical Functions. Cambridge University Press, Cambridge.

[17] Abramowitz, M. and Stegun, I.A. (1965) Handbook of Mathematical Functions with Formulas, Graphs, and Mathematical Tables. Dover, New York.

[18] Schechter, P. (1976) An Analytic Expression for the Luminosity Function for Galaxies. ApJ, 203, 297. https://doi.org/10.1086/154079

[19] Press, W.H., Teukolsky, S.A., Vetterling, W.T. and Flannery, B.P. (1992) Numerical Recipes in FORTRAN. The Art of Scientific Computing. Cambridge University Press, Cambridge.

[20] Akaike, H. (1974) A New Look at the Statistical Model Identification. IEEE Transactions on Automatic Control, 19, 716-723. https://doi.org/10.1109/TAC.1974.1100705

[21] Liddle, A.R. (2004) How Many Cosmological Parameters? MNRAS, 351, L49. 
https://doi.org/10.1111/j.1365-2966.2004.08033.x

[22] Godlowski, W. and Szydowski, M. (2005) Constraints on Dark Energy Models from Supernovae. In: Turatto, M., Benetti, S., Zampieri, L. and Shea, W., Eds., 1604-2004: Supernovae as Cosmological Lighthouses, Vol. 342 of Astronomical Society of the Pacific Conference Series, Astronomical Society of the Pacific, San Francisco, 508-516.

[23] Kolmogoroff, A. (1941) Confidence Limits for an Unknown Distribution Function. The Annals of Mathematical Statistics, 12, 461-463.

https://doi.org/10.1214/aoms/1177731684

[24] Smirnov, N. (1948) Table for Estimating the Goodness of Fit of Empirical Distributions. The Annals of Mathematical Statistics, 19, 279-281.

https://doi.org/10.1214/aoms/1177730256

[25] Massey Frank, J.J. (1951) The Kolmogorov-Smirnov Test for Goodness of Fit. Journal of the American Statistical Association, 46, 68-78. https://doi.org/10.1080/01621459.1951.10500769

[26] Zaninetti, L. (2020) New Probability Distributions in Astrophysics: II. The Generalized and Double Truncated Lindley. International Journal of Astronomy and Astrophysics, 10, 39-55. https://doi.org/10.4236/ijaa.2020.101004

[27] Blanton, M.R., Hogg, D.W., Bahcall, N.A., Brinkmann, J. and Britton, M. (2003) The Galaxy Luminosity Function and Luminosity Density at Redshift $z=0.1$. APJ, 592, 819. https://doi.org/10.1086/375776

[28] Zaninetti, L. (2017) A Left and Right Truncated Schechter Luminosity Function for Quasars. Galaxies, 5, 25-40. https://doi.org/10.3390/galaxies5020025

[29] Zaninetti, L. (2019) The Truncated Lindley Distribution with Applications in Astrophysics. Galaxies, 7, 61. https://doi.org/10.3390/galaxies7020061 